\title{
Influencia mutua de la deformación y composición química sobre la precipitación inducida en aceros microaleados
}

\author{
A. Quispe*, S.F. Medina* y M. Gómez* \\ Resumen Mediante ensayos de torsión y usando el método back extrapolation se ha determinado la \\ cinética de recristalización estática de aceros microaleados con vanadio $(\mathrm{V})$, niobio $(\mathrm{Nb})$ y \\ titanio ( $\mathrm{Ti}$ ) y, a partir de las mismas, ha sido posible dibujar los diagramas recristalización- \\ precipitación-tiempo-temperatura (RPTT), que muestran gráficamente la interacción \\ recristalización-precipitación. Estos diagramas muestran que el efecto de la deformación en \\ la cinética de precipitación depende del contenido de microaleante. En este sentido, se \\ propone una nueva expresión para relacionar la influencia de la deformación y del \\ contenido de microaleante sobre el periodo mínimo de incubación de la precipitación \\ inducida.
}

Palabras clave Deformación. Acero microaleado. Diagrama RPTT. Cinética de precipitación. Periodo de incubación.

\section{Influence mutual of the deformation and chemical composition on induced precipitation in microalloyed steels}

\begin{abstract}
By means of torsion tests and applying the "back extrapolation" method, the static recrystallization kinetics in microalloyed steels with vanadium $(\mathrm{V})$, niobium $(\mathrm{Nb})$ and titanium ( $\mathrm{Ti}$ ) has been determined and, recrystallization-precipitation-time-temperature (RPTT) diagrams have been plotted also graphically, which show the RecrystallizationPrecipitation interaction. These diagrams show that the effect of the deformation on the precipitation kinetics depends of the microalloy content. In this sense, a new expression is proposed to relate the influence of the deformation and the chemical composition on the minimum incubation of the precipitation kinetics.
\end{abstract}

Keywords Deformation. Microalloyed steel. RPTT diagram. Precipitation kinetics. Incubation time.

\section{INTRODUCCIÓN}

La recristalización estática de los aceros microaleados es diferente antes y después de la precipitación inducida por la deformación. En el primer caso, todos los elementos están en solución y la cinética de recristalización ocurre de la misma forma que en los aceros de baja aleación, donde los diferentes elementos de aleación contribuyen en mayor o menor

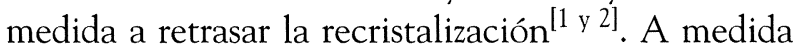
que la temperatura desciende se alcanza una temperatura crítica, a partir de la cual la recristalización estática es momentáneamente inhibida por efecto de la precipitación inducida por la deformación. Esta inhibición momentánea aparece como un plateau sobre las curvas de fracción recristalizada frente al tiempo. La buena definición del plateau permite trazar los diagramas de recristalización-precipitacióntiempo-temperatura (RPTT) y, consecuentemente, obtener un mayor conocimiento de cómo las condiciones de laminación influyen sobre la microestructura de estos aceros ${ }^{[3}$ y 4$]$. El plateau comienza a presentarse por debajo de una cierta temperatura, conocida como Temperatura Crítica de Recristalización Estática (SRCT). Hasta muy recientemente, se consideraba que la influencia de la deformación en la cinética de precipitación era independiente de otras variables como, p.ej., el contenido y naturaleza de los microaleantes ${ }^{[5]}$. Sin embargo, Medina et al. ${ }^{[6]}$, en trabajos previos llevados a cabo en

(*) Centro Nacional de Investigaciones Metalúrgicas (CENIM), Avenida Gregorio del Amo, 828040 Madrid, España. 
aceros microaleados con titanio, vanadio y niobio demostraron experimentalmente que existe una interdependencia de la deformación y el contenido de microaleante sobre el período de incubación de la precipitación inducida, adoptando una nueva expresión que difiere conceptualmente de la expresión teórica de Dutta y Sellars ${ }^{[5]}$.

Este trabajo representa una continuación de trabajos anteriores ${ }^{[6]}$ habiéndose utilizado más aceros en el estudio experimental y, por tanto, los resultados obtenidos cobran más firmeza.

\section{MATERIALES Y PROCEDIMIENTO EXPERIMEN- TAL}

Los aceros fueron fabricados por el proceso de refusión bajo electroescoria (ESR) en una unidad capaz de producir lingotes de $30 \mathrm{Kg}$. En la tabla I se muestra la composición química de los aceros estudiados. Las probetas usadas para torsión, tenían 50 $\mathrm{mm}$ de longitud útil y $3 \mathrm{~mm}$ de radio. Previamente a la deformación, las probetas eran austenizadas a temperaturas muy altas $\left(\geq 1.200^{\circ} \mathrm{C}\right)$ durante 10 min. A continuación, la temperatura era reducida rápidamente hasta la temperatura de ensayo. Se de- terminó el tamaño de grano austenítico a las temperaturas de austenización mediante la técnica de intersección lineal, analizando veinte campos para cada probeta (Tabla I). La técnica dilatométrica se usó para determinar la temperatura de transformación, $\mathrm{A}_{\mathrm{r} 3}$ (Tabla I). Conociendo estas temperaturas de transformación se eligieron temperaturas de ensayo que corresponden a la fase austenítica.

La fracción recristalizada se determinó utilizando el método conocido como back extrapolation para diferentes temperaturas, deformaciones equivalentes de 0,20 y 0,35 y una velocidad de deformación constante de $3,63 \mathrm{~s}^{-1}$ (=1.000 rev/min). Las deformaciones aplicadas se calcularon sobre la superficie cilíndrica de la probeta de acuerdo al criterio de Von Mises ${ }^{[7]}$.

\section{RESULTADOS Y DISCUSIÓN}

\subsection{Recristalización estática}

Algunas curvas de la fracción recristalizada $\left(\mathrm{X}_{\mathrm{a}}\right)$ frente al tiempo muestran la formación de un plateau, como ocurre en las curvas que corresponden a las temperaturas de $900^{\circ} \mathrm{C}, 850^{\circ} \mathrm{C}$ y $800^{\circ} \mathrm{C}$

Tabla I. Composición química de los aceros (\% peso); austenización $\left({ }^{\circ} \mathrm{C} \times 10 \mathrm{~min}\right)$; tamaño de grano (D); temperatura crítica de enfriamiento $\left(\mathrm{Ar}_{3}\right.$, a $0,20^{*}$ y $\left.0,25^{\circ} \mathrm{C} / \mathrm{s}\right)$

Table I. Chemical composition of the steels (mass \%); austenization $\left({ }^{\circ} \mathrm{C} \times 10 \mathrm{~min}\right.$ ); grain size (D); cooling critical temperature $\left(\mathrm{Ar}_{3}, a, 20^{*}\right.$ and $\left.0,25^{\circ} \mathrm{C} / \mathrm{s}\right)$

\begin{tabular}{|c|c|c|c|c|c|c|c|c|}
\hline Acero & $C$ & Sí & $M n$ & $\mathbf{N}$ & $\mathbf{X i}$ & $\begin{array}{l}\text { Austenización } \\
\left({ }^{\circ} \mathrm{C} \times 10 \mathrm{~min}\right)\end{array}$ & $\begin{array}{c}D \\
(\mu \mathrm{m})\end{array}$ & $\begin{array}{l}\mathrm{Ar}_{3} \\
\left({ }^{\circ} \mathrm{C}\right)\end{array}$ \\
\hline V1 & 0,11 & 0,24 & 1,10 & 0,0105 & $V=0,043$ & 1230 & 172 & $786^{*}$ \\
\hline V2 & 0,12 & 0,24 & 1,10 & 0,0123 & $V=0,060$ & 1230 & 167 & $782^{*}$ \\
\hline V3 & 0,11 & 0,24 & 1,00 & 0,0144 & $V=0,093$ & 1230 & 165 & $784^{*}$ \\
\hline V5 & 0,33 & 0,22 & 1,24 & 0,0146 & $V=0,076$ & 1200 & 165 & 716 \\
\hline V6 & 0,35 & 0,21 & 1,23 & 0,0121 & $V=0,033$ & 1200 & 170 & 715 \\
\hline V8 & 0,38 & 0,22 & 1,42 & 0,0190 & $V=0,120$ & 1200 & 157 & 721 \\
\hline $\mathrm{T} 1$ & 0,15 & 0,24 & 1,12 & 0,0105 & $\mathrm{Ti}=0,021$ & 1230 & 29 & $791^{*}$ \\
\hline $\mathrm{T} 2$ & 0,15 & 0,27 & 1,25 & 0,0100 & $\mathrm{Ti}=0,055$ & 1230 & 95 & $774^{*}$ \\
\hline T3 & 0,15 & 0,26 & 1,10 & 0,0102 & $\mathrm{Ti}=0,075$ & 1230 & 90 & $779 *$ \\
\hline $\mathrm{N} 1$ & 0,11 & 0,24 & 1,08 & 0,0058 & $\mathrm{Nb}=0,024$ & 1250 & 210 & $768^{*}$ \\
\hline N2 & 0,21 & 0,24 & 1,23 & 0,0112 & $\mathrm{Nb}=0,041$ & 1250 & 122 & $786^{*}$ \\
\hline N3 & 0,21 & 0,18 & 1,32 & 0,0119 & $\mathrm{Nb}=0,093$ & 1250 & 116 & 786 \\
\hline N4 & 0,21 & 0,19 & 1,14 & 0,0061 & $\mathrm{Nb}=0,058$ & 1250 & 190 & 679 \\
\hline N7 & 0,29 & 0,22 & 1,30 & 0,0062 & $\mathrm{Nb}=0,058$ & 1295 & 415 & 751 \\
\hline N8 & 0,20 & 0,20 & 1,00 & 0,0056 & $\mathrm{Nb}=0,007$ & 1250 & 140 & 770 \\
\hline N9 & 0,46 & 0,24 & 1,25 & 0,0100 & $\mathrm{Nb}=0,009$ & 1250 & 190 & 704 \\
\hline
\end{tabular}


Influencia mutua de la deformación y composición química sobre la precipitación inducida en aceros microaleados A. QUispe, S.F. MEDINA Y M. GÓMEZ

de la figura 1a) referidas al acero V5. Las curvas correspondientes a temperaturas mayores tienen la forma "sigmoidal" de la ley de Avrami. Cada plateau muestra que la precipitación no inhibe la recristalización permanentemente y, de nuevo, progresa hasta que se completa, siguiendo la forma "sigmoidal" registrada antes de la formación del mismo. Por tanto, antes y después de dicho plateau, la cinética de recristalización obedece a la ley de Avrami.

Los aceros microaleados con niobio, tales como N4 y N7, mostraron un doble plateau (Fig. 1b), como puede observarse en las curvas correspondientes a $1.050^{\circ} \mathrm{C}$ y $1.000{ }^{\circ} \mathrm{C}$. Este fenómeno ha sido interpretado como la precipitación de dos tipos de carbonitruros de niobio, de estequiometría algo diferente, que comienzan a formarse, prácticamente, a la misma temperatura. Una vez que los precipitados formados sobre el primer plateau han alcanzado un cierto tamaño crítico debido al crecimiento por nucleación heterogénea y a la coalescencia entre ellos, las fuerzas de anclaje ejercidas por los mis-
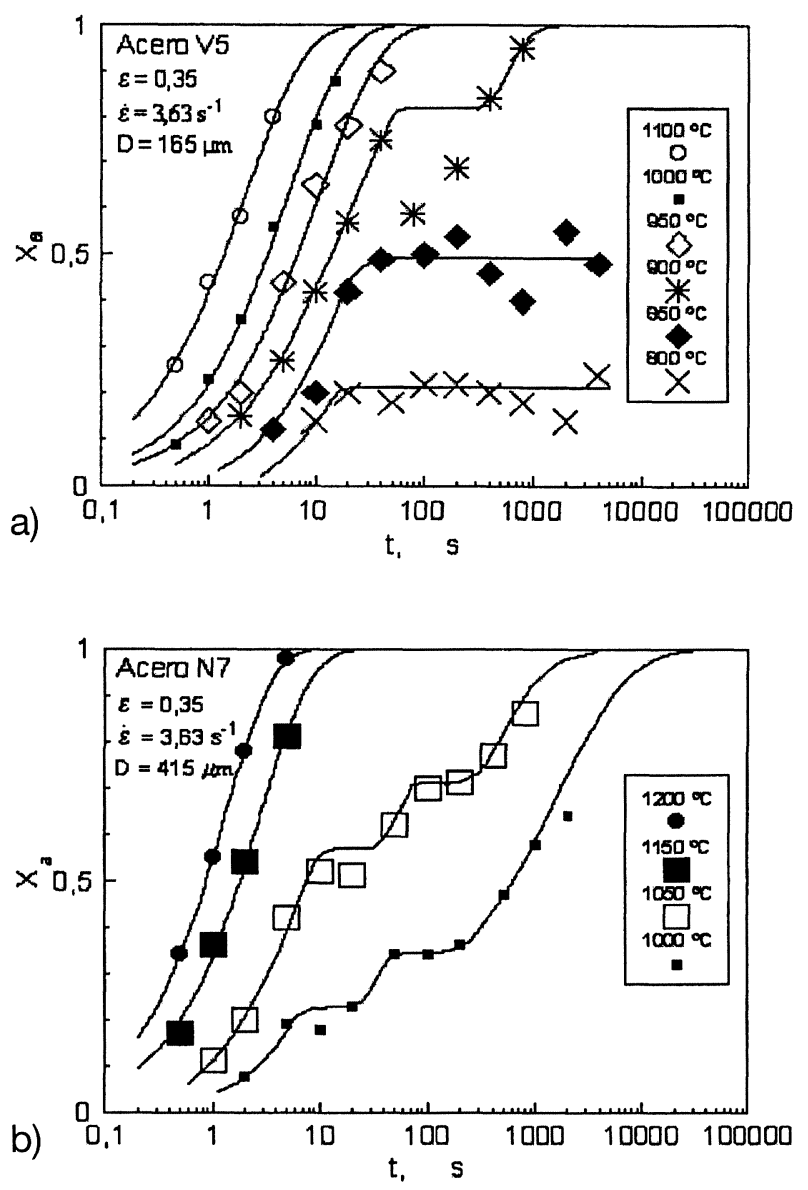

Figura 1. Fracción recristalizada $\left(X_{a}\right)$ frente al tiempo $(t)$. Acero: (a) V5 y (b) N7.

Figure 1. Recrystallized fraction $\left(X_{a}\right)$ against the time $(t)$. Steel: (a) V5 and (b) N7. mos disminuyen lo suficiente como para permitir que la recristalización progrese, de nuevo, hasta que comienza a producirse la segunda precipitación ${ }^{[8]}$. El segundo plateau muestra la segunda precipitación y el ciclo se repite hasta que, nuevamente, la recristalización continúa hasta completarse ${ }^{[9]}$.

\subsection{Diagramas RPTT}

De las curvas de fracción recristalizada $\left(\mathrm{X}_{\mathrm{a}}\right)$ frente al tiempo, se determinaron los diagramas RPTT mediante lectura de las temperaturas y tiempos correspondientes a los puntos de intersección de las líneas horizontales trazadas a diferentes fracciones recristalizadas $(0,1 ; 0,3 ; 0,5 ; 0,7 ; 0,9$ y 0,95$)$ con las curvas $\mathrm{X}_{\mathrm{a}} / \mathrm{t}$. De este modo, se dibujaron los diagramas RPTT como se muestran en la figura 2, a) y b) (aceros V8 y N4). Se observa que la fracción recristalizada no varía entre las curvas de inicio $\left(\mathrm{P}_{\mathrm{s}}\right)$ y final $\left(\mathrm{P}_{\mathrm{f}}\right)$ de la precipitación y está representada por una línea horizontal. Una vez que las líneas de cada fracción recristalizada han alcanzado la curva $\mathrm{P}_{\mathrm{f}}$, continúan descendiendo con
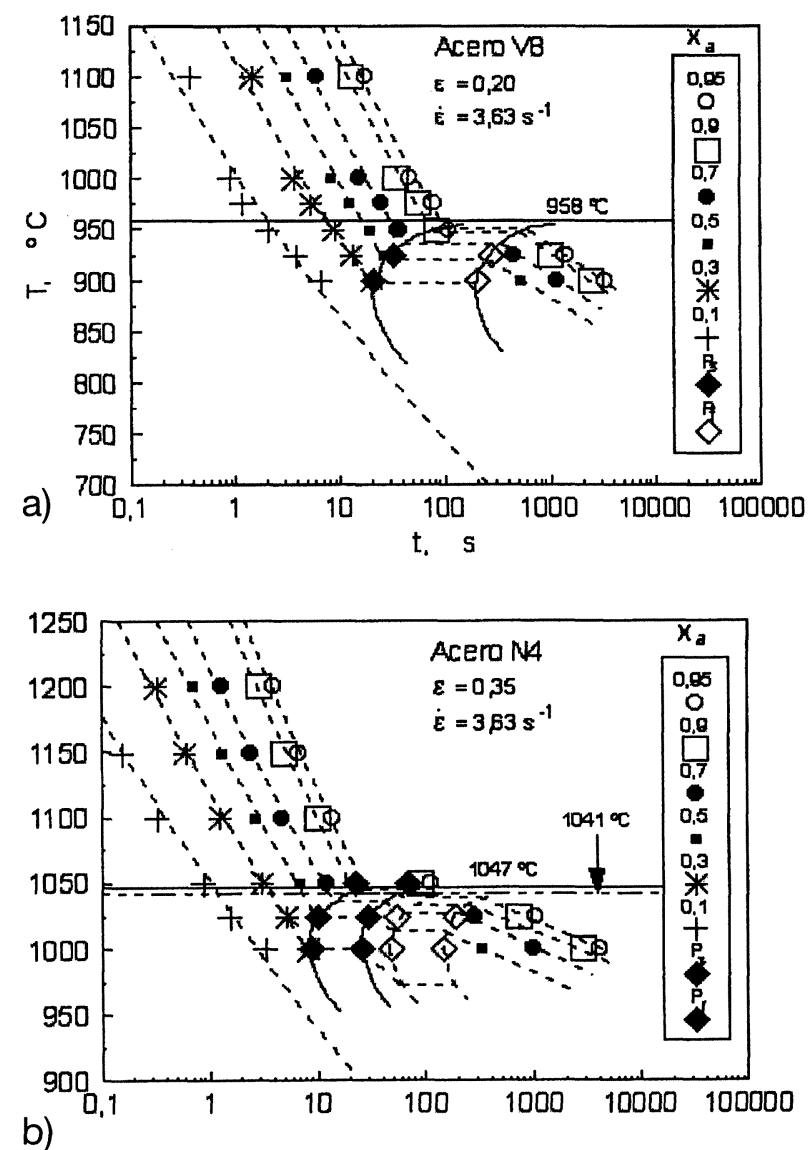

Figura 2. Diagrama RPTT. Aceros: (a) V8 y (b) N4.

Figure 2. RPTT diagram. Steel: (a) V8 and (b) N4. 
menor pendiente que antes de alcanzar $\mathrm{P}_{\mathrm{s}}$. Los diagramas RPTT proporcionan una valiosa información sobre la interacción recistalización-precipitación. Por ejemplo, se conoce el instante aproximado de comienzo de la precipitación a cualquier temperatura (curva $\mathrm{P}_{\mathrm{s}}$ ), el tiempo mínimo de incubación, dado por la nariz de la curva $\mathrm{P}_{s}$, el instante final de la precipitación (curva $\mathrm{P}_{\mathrm{f}}$ ), la fracción recristalizada que corresponde a cualquier punto de la curva $\mathrm{P}_{s}$, etc. Es interesante resaltar que la nariz de la curva $\mathrm{P}_{\mathrm{s}}$ intersecta con la línea de, aproximadamente, un 0,5 de fracción recristalizada, es decir, en ese punto, el volumen recristalizado es del
$50 \%$. Asimismo, cuando el volumen recristalizado es menor del $20 \%$ la nucleación de los precipitados es prácticamente imposible ya que la temperatura es excesivamente baja para que se produzca la difusión de los elementos microaleantes y la correspondiente precipitación.

\subsection{Influencia de la deformación y los elemen- tos aleantes}

Los valores del tiempo mínimo de incubación $\left(t_{N}\right)$ y del tiempo mínimo del final de la precipitación $\left(t^{\prime}\right)$ se muestran en tabla II: Se observa que los

Tabla II. Tiempo mínimo de incubación $t_{N}, \beta$ experimental y calculado

Table II. Minimum incubation time $t_{N}, \beta$ experimental and calculated

\begin{tabular}{|c|c|c|c|c|c|c|c|}
\hline Acero & $\varepsilon$ & $t_{\mathrm{N} 1},(s)$ & $\mathbf{t}_{\mathrm{N} 1}^{\prime},(\mathrm{s})$ & $\mathrm{t}_{\mathrm{N} 2},(\mathrm{~s})$ & $\mathrm{t}_{\mathrm{N} 2}^{\prime}(\mathrm{s})$ & $\beta_{\exp }$ & $\beta_{\mathrm{cal}}$ \\
\hline \multirow{2}{*}{ V1 } & 0,20 & 52 & 340 & - & - & 0,983 & 0,790 \\
\hline & 0,35 & 30 & 200 & - & - & & \\
\hline \multirow{2}{*}{ V2 } & 0,20 & 37 & 330 & - & - & 0,699 & 0,637 \\
\hline & 0,35 & 24 & 155 & - & - & & \\
\hline \multirow{2}{*}{ V3 } & 0,20 & 20 & 120 & - & - & 0,400 & 0,472 \\
\hline & 0,35 & 16 & 115 & - & - & & \\
\hline \multirow{2}{*}{ V5 } & 0,20 & 21 & 180 & - & - & 0,543 & 0,544 \\
\hline & 0,35 & 16 & 110 & - & - & & \\
\hline \multirow{2}{*}{ V6 } & 0,20 & 40 & 80 & - & - & 1,006 & 0,978 \\
\hline & 0,35 & 23 & 41 & - & - & & \\
\hline \multirow{2}{*}{ V8 } & 0,20 & 19 & 180 & - & - & 0,422 & 0,393 \\
\hline & 0,35 & 15 & 90 & - & - & & \\
\hline \multirow{2}{*}{$\mathrm{T} 1$} & 0,20 & 300 & 1800 & - & - & 1,960 & 1,904 \\
\hline & 0,35 & 100 & 600 & - & - & & \\
\hline \multirow{2}{*}{$\mathrm{T} 2$} & 0,20 & 130 & 300 & - & - & 1,710 & 1,104 \\
\hline & 0,35 & 50 & 220 & - & - & & \\
\hline \multirow{2}{*}{ T3 } & 0,20 & 60 & 150 & - & - & 0,330 & 0,801 \\
\hline & 0,35 & 50 & 150 & - & - & & \\
\hline \multirow{2}{*}{$\mathrm{N} 1$} & 0,20 & 24 & 295 & - & - & 0,770 & 0,813 \\
\hline & 0,35 & 16 & 190 & - & - & & \\
\hline \multirow{2}{*}{ N2 } & 0,20 & 17 & 275 & - & - & 0,580 & 0,472 \\
\hline & 0,35 & 12 & 130 & - & - & & \\
\hline \multirow{2}{*}{ N3 } & 0,20 & 26 & 460 & - & - & 0,983 & 1,011 \\
\hline & 0,35 & 15 & 200 & - & - & & \\
\hline \multirow{2}{*}{ N4 } & 0,20 & 11 & 38 & 80 & 230 & 0,592 & 0,652 \\
\hline & 0,35 & 8 & 23 & 42 & 130 & & \\
\hline \multirow{2}{*}{ N7 } & 0,20 & 10 & 35 & 75 & 215 & 0,637 & 0,598 \\
\hline & 0,35 & 7 & 20 & 42 & 110 & & \\
\hline \multirow{2}{*}{ N8 } & 0,20 & 58 & 510 & - & - & 1,653 & 1,755 \\
\hline & 0,35 & 23 & 220 & - & - & & \\
\hline \multirow{2}{*}{ N9 } & 0,20 & 29 & 82 & - & - & 1,502 & 1,649 \\
\hline & 0,35 & 12 & 38 & - & - & & \\
\hline
\end{tabular}


tiempos $t_{N}$ y $t^{\prime}{ }_{N}$ disminuyen con el aumento del contenido de microaleante y deformación. Sin embargo, puede apreciarse que las dos magnitudes no actúan independientemente, y el efecto de la deformación sobre la cinética de precipitación depende del contenido de microaleante. La forma de las curvas RPTT muestran que el modelo establecido por Dutta et al. ${ }^{[5 \text { y } 10]}$ es, en general, cualitativamente correcto. Dichos autores establecen que el tiempo, $t_{0,05}$, correspondiente a una precipitación parcial de $5 \%$ está relacionado con el contenido de microaleante $(w)$ y deformación $(\varepsilon)$ de acuerdo con la siguiente expresión:

$$
t_{0,05} \infty w^{-v} \varepsilon^{-\beta}
$$

De acuerdo con la ecuación (1) y aceptando que $t_{0,05}$ y $t_{N}$ son aproximadamente similares, es decir, se refieren al $5 \%$ de volumen precipitado, los valores de $\beta$ fueron determinados y los resultados se muestran en la tabla II.

Se observa que $\beta$ disminuye con el incremento del contenido de microaleante, en cada grupo de aceros. La representación gráfica de $\beta$ frente al contenido (\% peso) del microaleante (Fig. 3), muestra claramente la dependencia de $\beta$. La forma de la curva de regresión sugiere una expresión de tipo Avrami para $\beta$, como sigue:

$$
\beta=1,96\left[1-\exp \left(-3,994 \times 10^{-2}\left(\frac{1}{w}\right)^{0,813}\right)\right]
$$

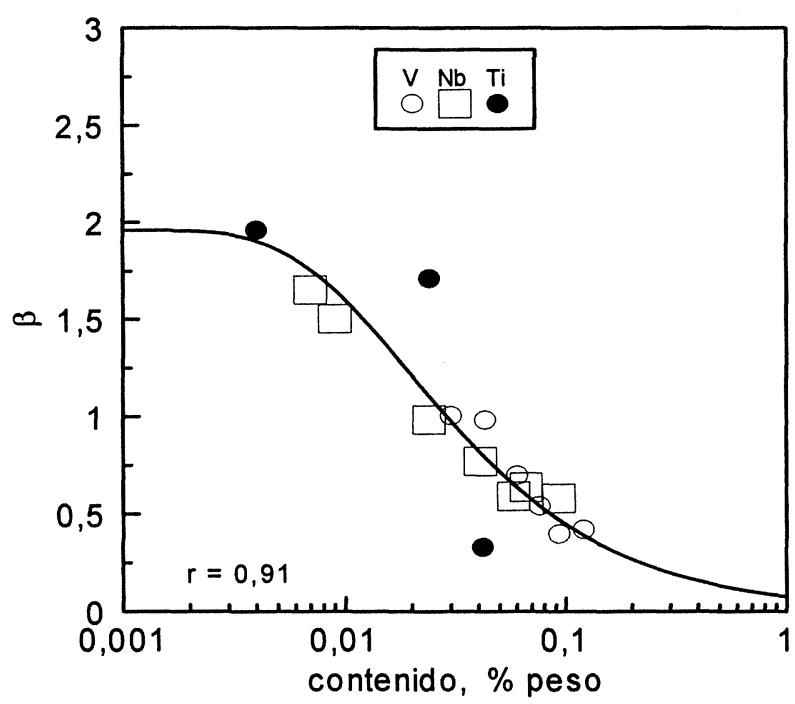

Figura 3. Parámetro $b$ frente al contenido de $\mathrm{V}, \mathrm{Nb}$ y Ti $(\%$ peso) para los aceros usados.

Figure 3. Parameter $b$ against the content of $V, N b$ and $T i$ (mass \%) for the steels used. donde, $w$ es el contenido de microaleante en $\%$ en peso.

Cálculos realizados con la ecuación (2) sugieren que la deformación inicial influye en la cinética de precipitación cuando el contenido de microaleante es menor a una cierta cantidad y que, en términos prácticos, debe ser, aproximadamente, $0,3 \%$ en peso. Para contenidos inferiores, el valor de $\beta$ debe ser mayor que cero y, consecuentemente, la deformación afectará a la cinética de precipitación. Uno de los efectos de la deformación es el incremento de la densidad de dislocaciones, que son fuentes de nucleación para los precipitados y este efecto se debilita gradualmente a medida que aumenta el porcentaje de microaleante, o sea, su actividad termodinámica. En la tabla II se comparan los valores experimentales de $\beta$ con los calculados de acuerdo con la ecuación (2).

Por último, dado que los precipitados son potenciales fuentes de nucleación de la ferrita ${ }^{[11]}$, especialmente en aceros microaleados con vanadio, sería interesante conocer el papel que juega el parámetro $\beta$ sobre dicha nucleación intragranular.

Además, el trabajo mostrado supone una parte de los trabajos realizados en el contexto de un proyecto financiado por CECA, cuyo objetivo fue el estudio de la interacción recristalización-precipitación en aceros microaleados ${ }^{[12]}$.

\section{CONCLUSIONES}

- La influencia de la deformación en el período de incubación para la precipitación disminuye cuando el contenido de microaleante se incrementa.

- Para propósitos prácticos se debe considerar que para contenidos mayores a $0,3 \%$ en peso la deformación no tiene influencia en la cinética de precipitación.

- Cuando el contenido de elemento microaleante disminuye el parámetro $b$ incrementa, pero no supera el valor de 2 .

\section{Agradecimientos}

Los autores desean agradecer a la Secretaría de Estado de Educación y Universidades por financiar la estancia en el CENIM del Dr. A. Quispe.

\section{REFERENCIAS}

[1] O. Kwon, ISIJ Int. 32 (1992) 350-358. 
[2] A. QuisPe, S.F. Medina y P. Valles, ISIJ Int. 37 (1997) 783-788

[3] S.F. Medina y A. Quispe, ISIJ Int. 41 (2001) 774-781.

[4] R. Lagneborg, T. Siwecki, S. Zajac y B. Hutchinson, Scand. J. Metall. 28 (1999) 186-241.

[5] B. DutTa y C.M. Sellars, Mater. Sci. Technol. 3 (1987) 197-207.

[6] S.F. MEdina y A. Quispe, ISIJ Int. 36 (1996) 1.295-1.300.

[7] A. FAESSEL, Rev. Metall. CIT 4 (1976) 875-892.

[8] S.F. Medina, M. Chapa, M. Gómez, A. Quispe, V. López y B. Fernández, Rev. Metal. Madrid 39 (2003) 403-407.
[9] S.F. MedinA, A. Quispe y M. Gómez, ISIJ Int. 41 (2001) 774-781.

[10] B. Dutta, E.J. Palmiere y C.M. Sellars, Acta Metall. Mater. 49 (2001) 785-794.

[11] C. Capdevila, F.G. Caballero y C. García de Andrés, Rev. Metal. Madrid 38 (2002) 183-194.

[12] S.E. Medina, F. López y M. Morcillo, Rev. Metal. Madrid 39 (2003) 193-204. 